\title{
The Convergence of Riemann Solutions to the Modified Chaplygin Gas Equations with a Coulomb-Like Friction Term as the Pressure Vanishes
}

\author{
Yongqiang Fan, ${ }^{1}$ Lihui Guo $\mathbb{D},{ }^{1}$ and Gan Yin $^{2}$ \\ ${ }^{1}$ College of Mathematics and System Sciences, Xinjiang University, Urumqi 830046, China \\ ${ }^{2}$ Department of Mathematics, Zhejiang University of Science \& Technology, Hangzhou, Zhejiang 310023, China
}

Correspondence should be addressed to Lihui Guo; lihguo@126.com

Received 28 May 2018; Accepted 17 July 2018; Published 1 August 2018

Academic Editor: Claudio Dappiaggi

Copyright (C) 2018 Yongqiang Fan et al. This is an open access article distributed under the Creative Commons Attribution License, which permits unrestricted use, distribution, and reproduction in any medium, provided the original work is properly cited.

\begin{abstract}
This paper studies the convergence of Riemann solutions to the inhomogeneous modified Chaplygin gas equations as the pressure vanishes. The delta shock waves and vacuum states occur as the pressure vanishes. The Riemann solutions of inhomogeneous modified Chaplygin gas equations are no longer self-similar. It is obviously different from the Riemann solutions of homogeneous modified Chaplygin gas equations. When the pressure vanishes, the Riemann solutions of the modified Chaplygin gas equations with a coulomb-like friction term converge to the Riemann solutions of the pressureless Euler system with a source term.
\end{abstract}

\section{Introduction}

The inhomogeneous modified Chaplygin gas equations have the following form:

$$
\begin{aligned}
\rho_{t}+(\rho u)_{x} & =0, \\
(\rho u)_{t}+\left(\rho u^{2}+P\right)_{x} & =\beta \rho,
\end{aligned}
$$

where $\beta$ is a constant and $\rho, u$ denote the density and the velocity, respectively. The scalar pressure $P=P(\rho, \alpha)$ is the modified Chaplygin gas pressure $P=\alpha p$ satisfying $\lim _{\alpha \rightarrow 0} P(\rho, \alpha)=0$, where $\alpha$ is a sufficiently small positive parameter. Meanwhile, the pressure $p$ satisfies the following equation of state:

$$
p=A \rho-\frac{B}{\rho}
$$

where $A>0, B>0$ are two positive constants. Modified Chaplygin gas (MCG) model was proposed in [1] by Benaoum in 2002. MCG $[2,3]$ represents the evaluation of the cosmology starting from the radiation era to the $\Lambda$ cold dark matter $(\Lambda \mathrm{CDM})$ model mentioned in [2-5]. As an exotic fluid, the MCG plays an important role in describing the accelerated expansion of the universe. In recent years, some researchers made some studies on the thermal equation of state to MCG and found that it could cool down in some constraints of parameters [6]. To know more interesting results related to MCG, the readers are referred to [7-12].

If $\beta=0$, the system (1) becomes the homogeneous modified Chaplygin gas equations. In [13], Yang and Wang considered the formation of delta shock waves and the vacuum states in the solutions of the homogeneous isentropic Euler equations for modified Chaplygin gas when the pressure vanishes.

Letting $A=0, B=1$ in (2), the equation of state is Chaplygin gas which was introduced by Chaplygin [14] in 1904, Tsien [15], and von Karman [16] as a suitable mathematical approximation for calculating the lifting force on a wing of an airplane in aerodynamics. The Chaplygin gas can be used to describe the dark energy. For the Riemann problem of homogeneous Chaplygin gas equations, there are lots of results. We refer the readers to [17-23]. For inhomogeneous Chaplygin gas equations, Shen [24] studied Riemann problem by introducing a new velocity:

$$
v(x, t)=u(x, t)-\beta t,
$$

which was introduced by Faccanoni and Mangeney in [25]. In 2016, Sun [26] studied the non-self-similar Riemann solution 
of inhomogeneous generalized Chaplygin gas equations. Guo, Li, Pan, and Han [27] considered the Riemann problem with delta initial data for the one-dimensional Chaplygin gas equations with a source term.

As $\alpha \longrightarrow 0$, system (1)-(2) becomes the inhomogeneous pressureless Euler system:

$$
\begin{gathered}
\rho_{t}+(\rho u)_{x}=0, \\
(\rho u)_{t}+\left(\rho u^{2}\right)_{x}=\beta \rho .
\end{gathered}
$$

Shen [28] considered the Riemann problem of pressureless Euler system (4). Daw and Nedeljkov [29] studied the shadow waves for pressureless gas balance laws.

In this paper, we are concerned with the convergence of the Riemann solutions to the inhomogeneous modified Chaplygin gas equations as the pressure vanishes. Firstly, we give the Riemann solutions of the inhomogeneous modified Chaplygin gas equations. Then, we study the convergence of the Riemann solutions to the modified Chaplygin gas equations with a source term as the pressure vanishes. We find that the Riemann solutions of inhomogeneous modified Chaplygin gas equations converge to the corresponding Riemann solutions of the pressureless Euler system. We mainly use the method of vanishing pressure limits which was introduced by $\mathrm{Li}[30]$ and Chen and Liu [31, 32] in which they studied the formation of delta shocks and vacuum states in the vanishing pressure limit of solutions to the isentropic Euler equations and the concentration and cavitation in the vanishing pressure limit of solutions to the Euler equations for nonisentropic fluids. Shen [33] considered the limits of Riemann solutions to the isentropic magnetogasdynamics. Shen and Sun [34] studied the formation of delta shocks and vacuum states in the vanishing pressure limit of Riemann solutions to the perturbed Aw-Rascle model. Sheng, Wang, and Yin [35] studied the vanishing pressure limit of the generalized Chaplygin gas dynamics system. Yin and Sheng [36] considered the delta shocks and vacuum states in vanishing pressure limit of solutions to the relativistic Euler equations for polytropic gases. For inhomogeneous equations, Guo, Li, and Yin $[37,38]$ considered the vanishing pressure limits of Riemann solutions to the Chaplygin gas equations with a source term and the limit behavior of the Riemann solutions to the generalized Chaplygin gas equations with a source term.

We organize this article as follows: in Section 2, we give some preliminaries which include the consideration of Riemann solutions to system (1)-(2) and the review of Riemann solutions to system (4). In Section 3, we study the convergence of Riemann solutions to system (1)-(2).

\section{Some Preliminaries}

In this section, we consider the Riemann solutions of the inhomogeneous modified Chaplygin gas equations and briefly review the Riemann solutions of pressureless Euler system with a coulomb-like friction term.

2.1. Riemann Problem for (1)-(2). In this subsection, we are concerned with the Riemann problem of (1)-(2).
From (3), system (1)-(2) is turned into the following conservation laws:

$$
\begin{array}{r}
\rho_{t}+(\rho(v+\beta t))_{x}=0, \\
(\rho v)_{t}+\left(\rho v(v+\beta t)+\alpha\left(A \rho-\frac{B}{\rho}\right)\right)_{x}=0 .
\end{array}
$$

We consider the Riemann initial value as follows:

$$
(v, \rho)(x, 0)=\left(u_{ \pm}, \rho_{ \pm}\right), \quad \pm x \geq 0,
$$

where $\rho_{ \pm} \geq 0$ and $u_{ \pm}$are given constants. Letting $f(\rho, \alpha)=$ $\sqrt{\alpha\left(A+B / \rho^{2}\right)}$, the two eigenvalues of system (5) are $\lambda_{1}=$ $v+\beta t-f(\rho, \alpha), \lambda_{2}=v+\beta t+f(\rho, \alpha)$. For arbitrary positive constants $A, B$, system (5) is strictly hyperbolic. The corresponding right eigenvectors are $\vec{r}_{1}=(-\rho, f(\rho, \alpha))^{T}$, $\vec{r}_{2}=(\rho, f(\rho, \alpha))^{T}$. By simple calculation, we obtain that $\nabla \lambda_{i} \cdot \vec{r}_{i}=A \alpha / f(\rho, \alpha) \neq 0,(i=1,2)$, which implies that both the characteristic fields are genuinely nonlinear.

Given a state $\left(u_{-}, \rho_{-}\right)$in the phase plane, the curve of backward rarefaction wave is

$$
R_{1}^{\alpha}\left(u_{-}, \rho_{-}\right):\left\{\begin{array}{l}
\frac{d x}{d t}=\lambda_{1}=v+\beta t-f(\rho, \alpha), \\
v=u_{-}-g\left(\rho_{-}, \rho, \alpha\right), \\
\lambda_{1}\left(u_{-}, \rho_{-}\right)<\lambda_{1}(v, \rho), \\
\quad \rho<\rho_{-}, v>u_{-},
\end{array}\right.
$$

and the corresponding curve of forward rarefaction wave is

$$
R_{2}^{\alpha}\left(u_{-}, \rho_{-}\right):\left\{\begin{array}{l}
\frac{d x}{d t}=\lambda_{2}=v+\beta t+f(\rho, \alpha), \\
v=u_{-}+g\left(\rho_{-}, \rho, \alpha\right), \\
\lambda_{2}\left(u_{-}, \rho_{-}\right)<\lambda_{2}(v, \rho), \\
\rho>\rho_{-}, v>u_{-},
\end{array}\right.
$$

where

$$
\begin{aligned}
& g\left(\rho_{-}, \rho, \alpha\right)=\int_{\rho_{-}}^{\rho} \frac{f(\rho, \alpha)}{\rho} d \rho=-f(\rho, \alpha)+f\left(\rho_{-}, \alpha\right) \\
& \quad+\sqrt{A \alpha}(\ln (f(\rho, 1)+\sqrt{A})+\ln \rho \\
& \left.\quad-\ln \left(f\left(\rho_{-}, 1\right)+\sqrt{A}\right)-\ln \rho_{-}\right) .
\end{aligned}
$$

Denote the propagating speed of the bounded discontinuity $x=x(t)$ as $\sigma(t)=x^{\prime}(t)$. The Rankine-Hugoniot conditions read

$$
\begin{aligned}
-\sigma(t)[\rho]+[\rho(v+\beta t)] & =0, \\
-\sigma(t)[\rho v]+\left[\rho v(v+\beta t)+\alpha\left(A \rho-\frac{B}{\rho}\right)\right] & =0 .
\end{aligned}
$$

For convenience, we write $h\left(\rho, \rho_{-}, \alpha\right)=\sqrt{\left(\alpha / \rho \rho_{-}\right)\left(A+B / \rho \rho_{-}\right)}$. Then for a given state $\left(u_{-}, \rho_{-}\right)$, from $(10)$, we obtain two kinds of shock wave curves, i.e., the backward shock wave curve, 


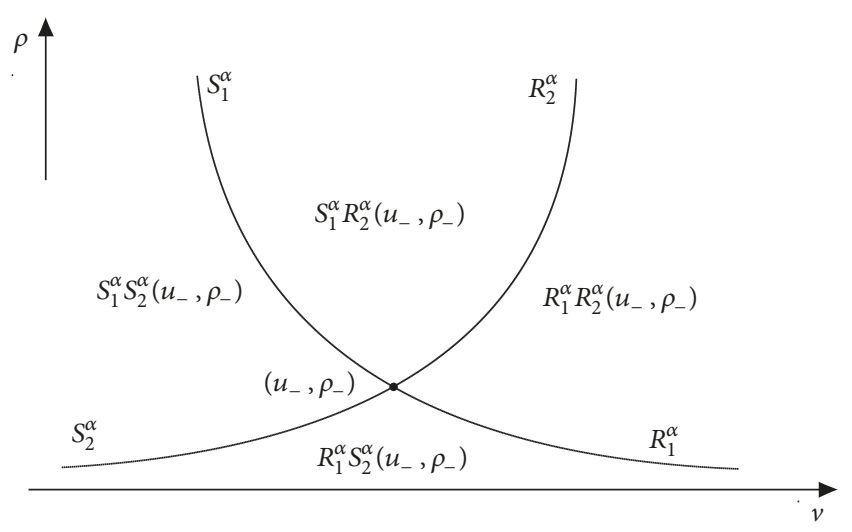

FIGURE 1: The phase plane $(v, \rho)$.

$$
S_{1}^{\alpha}\left(u_{-}, \rho_{-}\right):\left\{\begin{array}{l}
\sigma_{1}^{\alpha}(t)=u_{-}+\beta t-\rho h\left(\rho, \rho_{-}, \alpha\right), \\
v=u_{-}-h\left(\rho, \rho_{-}, \alpha\right)\left(\rho-\rho_{-}\right),
\end{array}\right.
$$

$$
\rho>\rho_{-}, v<u_{-},
$$

and the forward shock wave curve,

$$
S_{2}^{\alpha}\left(u_{-}, \rho_{-}\right):\left\{\begin{array}{l}
\sigma_{2}^{\alpha}(t)=u_{-}+\beta t+\rho h\left(\rho, \rho_{-}, \alpha\right), \\
v=u_{-}+h\left(\rho, \rho_{-}, \alpha\right)\left(\rho-\rho_{-}\right), \\
\rho<\rho_{-}, v<u_{-} .
\end{array}\right.
$$

In the phase plane, given a state $\left(u_{-}, \rho_{-}\right)$, the curves of $R_{j}^{\alpha}\left(u_{-}, \rho_{-}\right)$and $S_{j}^{\alpha}\left(u_{-}, \rho_{-}\right),(j=1,2)$ divide the phase plane into four regions.

When $\left(u_{+}, \rho_{+}\right) \in\left(R_{1}^{\alpha} R_{2}^{\alpha} \cup R_{1}^{\alpha} S_{2}^{\alpha} \cup S_{1}^{\alpha} R_{2}^{\alpha} \cup S_{1}^{\alpha} S_{2}^{\alpha}\right)\left(u_{-}, \rho_{-}\right)$(see Figure 1), the Riemann solutions of system (5) are

(1) $\left(u_{+}, \rho_{+}\right) \in R_{1}^{\alpha} R_{2}^{\alpha}\left(u_{-}, \rho_{-}\right):\left(u_{-}, \rho_{-}\right)+R_{1}^{\alpha}+\left(v_{* \alpha}, \rho_{* \alpha}\right)+$ $R_{2}^{\alpha}+\left(u_{+}, \rho_{+}\right)$

(2) $\left(u_{+}, \rho_{+}\right) \in R_{1}^{\alpha} S_{2}^{\alpha}\left(u_{-}, \rho_{-}\right):\left(u_{-}, \rho_{-}\right)+R_{1}^{\alpha}+\left(v_{* \alpha}, \rho_{* \alpha}\right)+$ $S_{2}^{\alpha}+\left(u_{+}, \rho_{+}\right)$

(3) $\left(u_{+}, \rho_{+}\right) \in S_{1}^{\alpha} R_{2}^{\alpha}\left(u_{-}, \rho_{-}\right):\left(u_{-}, \rho_{-}\right)+S_{1}^{\alpha}+\left(v_{* \alpha}, \rho_{* \alpha}\right)+$ $R_{2}^{\alpha}+\left(u_{+}, \rho_{+}\right)$

(4) $\left(u_{+}, \rho_{+}\right) \in S_{1}^{\alpha} S_{2}^{\alpha}\left(u_{-}, \rho_{-}\right):\left(u_{-}, \rho_{-}\right)+S_{1}^{\alpha}+\left(v_{* \alpha}, \rho_{* \alpha}\right)+$ $S_{2}^{\alpha}+\left(u_{+}, \rho_{+}\right)$,

where $\left(v_{* \alpha}, \rho_{* \alpha}\right)$ denotes the intermediate state. By using (3), we obtain the Riemann solutions of (1)-(2) as follows:

(1) $\left(u_{+}, \rho_{+}\right) \in R_{1}^{\alpha} R_{2}^{\alpha}\left(u_{-}, \rho_{-}\right):\left(u_{-}+\beta t, \rho_{-}\right)+R_{1}^{\alpha}+\left(v_{* \alpha}+\right.$ $\left.\beta t, \rho_{* \alpha}\right)+R_{2}^{\alpha}+\left(u_{+}+\beta t, \rho_{+}\right)$;

(2) $\left(u_{+}, \rho_{+}\right) \in R_{1}^{\alpha} S_{2}^{\alpha}\left(u_{-}, \rho_{-}\right):\left(u_{-}+\beta t, \rho_{-}\right)+R_{1}^{\alpha}+\left(v_{* \alpha}+\right.$ $\left.\beta t, \rho_{* \alpha}\right)+S_{2}^{\alpha}+\left(u_{+}+\beta t, \rho_{+}\right)$;

(3) $\left(u_{+}, \rho_{+}\right) \in S_{1}^{\alpha} R_{2}^{\alpha}\left(u_{-}, \rho_{-}\right)\left(u_{-}+\beta t, \rho_{-}\right)+S_{1}^{\alpha}+\left(v_{* \alpha}+\right.$ $\left.\beta t, \rho_{* \alpha}\right)+R_{2}^{\alpha}+\left(u_{+}+\beta t, \rho_{+}\right)$

(4) $\left(u_{+}, \rho_{+}\right) \in S_{1}^{\alpha} S_{2}^{\alpha}\left(u_{-}, \rho_{-}\right)\left(u_{-}+\beta t, \rho_{-}\right)+S_{1}^{\alpha}+\left(v_{* \alpha}+\right.$ $\left.\beta t, \rho_{* \alpha}\right)+S_{2}^{\alpha}+\left(u_{+}+\beta t, \rho_{+}\right)$.
2.2. Riemann Problem for (4). In this subsection, we restate the results on the Riemann solutions to system (4). The detailed results can be referred to in [28].

(1) For $u_{-}>u_{+}$, the Riemann solution of system (4) has the following form:

$$
\begin{aligned}
& \rho(x, t)= \begin{cases}\rho_{-}, & x<x(t), \\
\omega(t) \delta(x-x(t)), & x=x(t), \\
\rho_{+}, & x>x(t),\end{cases} \\
& u(x, t)= \begin{cases}u_{-}, & x<x(t), \\
u_{\delta}(t), & x=x(t), \\
u_{+}, & x>x(t),\end{cases}
\end{aligned}
$$

in which the delta shock wave is introduced into the Riemann solution (13)-(14). About the definition of delta shock wave, please refer to $[31,34,39,40]$, for example. The delta shock wave satisfies the generalized Rankine-Hugoniot conditions:

$$
\begin{aligned}
\frac{d x(t)}{d t} & =u_{\delta}(t), \\
\frac{d \omega(t)}{d t} & =u_{\delta}(t)[\rho]-[\rho u], \\
\frac{d\left(\omega(t) u_{\delta}(t)\right)}{d t} & =u_{\delta}(t)[\rho u]-\left[\rho u^{2}\right]+\beta \omega(t),
\end{aligned}
$$

where $x(t), \omega(t)$, and $u_{\delta}(t)$, respectively, denote the location, weight, and the velocity of delta shock wave, and $(x(0), w(0))=(0,0)$.

Letting $v_{\delta}=\left(\sqrt{\rho_{-}} u_{-}+\sqrt{\rho_{+}} u_{+}\right) /\left(\sqrt{\rho_{-}}+\sqrt{\rho_{+}}\right)$, by simple calculation, we obtain

$$
\begin{aligned}
\omega(t) & =\sqrt{\rho_{-} \rho_{+}}\left(u_{-}-u_{+}\right) t, \\
u_{\delta}(t) & =v_{\delta}+\beta t, \\
x(t) & =v_{\delta} t+\frac{1}{2} \beta t^{2},
\end{aligned}
$$

for $[\rho] \neq 0$ and

$$
\begin{aligned}
\omega(t) & =-[\rho u] t, \\
u_{\delta}(t) & =\frac{1}{2}\left(u_{+}+u_{-}\right)+\frac{1}{3} \beta t, \\
x(t) & =\frac{1}{2}\left(u_{+}+u_{-}\right) t+\frac{1}{6} \beta t^{2},
\end{aligned}
$$

for $[\rho]=0$.

(2) For $u_{-}<u_{+}$, the structure of Riemann solution is

$$
\left(u_{-}+\beta t, \rho_{-}\right)+J_{1}+V a c+J_{2}+\left(u_{+}+\beta t, \rho_{+}\right) .
$$

(3) For $u_{-}=u_{+}$, the structure of Riemann solution is

$$
\left(u_{-}+\beta t, \rho_{-}\right)+J^{\star}+\left(u_{+}+\beta t, \rho_{+}\right) .
$$




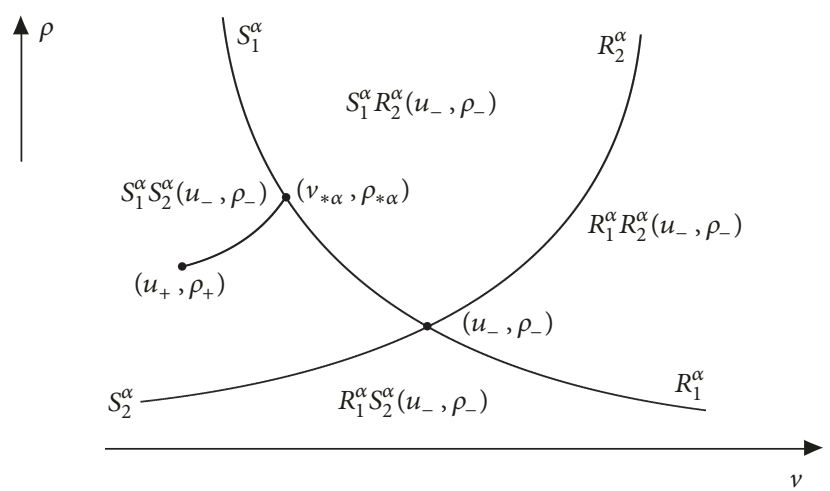

(a) The phase plane

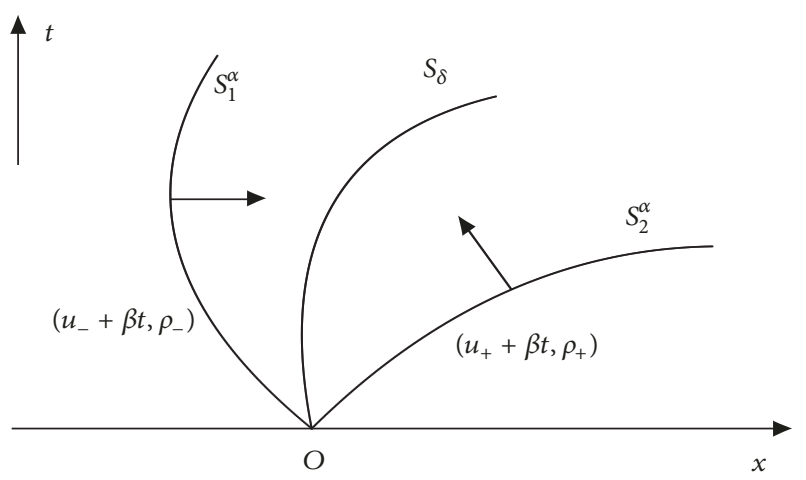

(b) The $(x, t)$ plane

FIgURE 2: Riemann solution when $\left(u_{+}, \rho_{+}\right) \in S_{1}^{\alpha} S_{2}^{\alpha}\left(u_{-}, \rho_{-}\right)$.

\section{Convergence of Riemann Solutions to (1)-(2)}

In this section, we consider the convergence of the Riemann solutions to system (1)-(2) in the vanishing pressure. We divide our discussions into three parts: the concentration and delta shock wave for $u_{-}>u_{+}$, the occurrence of vacuum for $u_{-}<u_{+}$, and the formation of discontinuity for $u_{-} \leq u_{+}$.

3.1. Concentration and Delta Shock Wave as the Pressure Vanishes. In this subcase, we show the phenomenon of the concentration and delta shock wave in the vanishing pressure limit of Riemann solutions to (1)-(2) when $u_{-}>u_{+}$(see Figure 2(a)).

Lemma 1. Assume $u_{-}>u_{+}$, then there exists certain value $\alpha_{1}>0$, such that $\left(u_{+}, \rho_{+}\right) \in S_{1}^{\alpha} S_{2}^{\alpha}\left(u_{-}, \rho_{-}\right)$when $0<\alpha<\alpha_{1}$.

Proof. If $u_{-}>u_{+}$and $\left(u_{+}, \rho_{+}\right) \in S_{1}^{\alpha} S_{2}^{\alpha}\left(u_{-}, \rho_{-}\right)$, we obtain (see Figure 2(a)):

$$
\begin{array}{ll}
u_{+}<u_{-}-h\left(\rho_{+}, \rho_{-}, \alpha\right)\left(\rho_{+}-\rho_{-}\right), & \rho_{+}>\rho_{-}, \\
u_{+}<u_{-}+h\left(\rho_{+}, \rho_{-}, \alpha\right)\left(\rho_{+}-\rho_{-}\right), & \rho_{+}<\rho_{-} .
\end{array}
$$

From (20)-(21) and (2), we have

$$
\sqrt{\alpha}\left|h\left(\rho_{+}, \rho_{-}, 1\right)\left(\rho_{+}-\rho_{-}\right)\right|<u_{-}-u_{+} .
$$

From (22), we derive that

$$
\alpha<\frac{\left(u_{-}-u_{+}\right)^{2}}{h^{2}\left(\rho_{+}, \rho_{-}, 1\right)\left(\rho_{+}-\rho_{-}\right)^{2}} .
$$

Let $\alpha_{1}=\left(u_{-}-u_{+}\right)^{2} / h^{2}\left(\rho_{+}, \rho_{-}, 1\right)\left(\rho_{+}-\rho_{-}\right)^{2}$, then $\left(u_{+}, \rho_{+}\right) \in$ $S_{1}^{\alpha} S_{2}^{\alpha}\left(u_{-}, \rho_{-}\right)$when $u_{-}>u_{+}$and $0<\alpha<\alpha_{1}$. It is obvious that this conclusion is also true for $\rho_{+}=\rho_{-}$, The proof is completed.
For $\left(u_{+}, \rho_{+}\right) \in S_{1}^{\alpha} S_{2}^{\alpha}\left(u_{-}, \rho_{-}\right)$, the Riemann solution of (5)(6) contains two shock waves satisfying

$$
\begin{aligned}
& S_{1}^{\alpha}:\left\{\begin{array}{l}
v_{* \alpha}=u_{-}-h\left(\rho_{* \alpha}, \rho_{-}, \alpha\right)\left(\rho_{* \alpha}-\rho_{-}\right), \\
\sigma_{1}^{\alpha}(t)=u_{-}+\beta t-\rho_{* \alpha} h\left(\rho_{* \alpha}, \rho_{-}, \alpha\right),
\end{array}\right. \\
& S_{2}^{\alpha}:\left\{\begin{array}{l}
v_{* \alpha}>\rho_{-}, \\
\sigma_{2}^{\alpha}(t)=u_{+}+h\left(\rho_{* \alpha}, \rho_{+}, \alpha\right)\left(\rho_{* \alpha}-\rho_{+}\right),
\end{array}\right. \\
& \rho_{* \alpha}>\rho_{+},
\end{aligned}
$$

where $\left(v_{* \alpha}, \rho_{* \alpha}\right)$ is the intermediate state (see Figure 2(a)). From the first equation of (24)-(25), we obtain

$$
\begin{aligned}
u_{-}-u_{+}= & h\left(\rho_{* \alpha}, \rho_{-}, \alpha\right)\left(\rho_{* \alpha}-\rho_{-}\right) \\
& +h\left(\rho_{* \alpha}, \rho_{+}, \alpha\right)\left(\rho_{* \alpha}-\rho_{+}\right), \quad \rho_{* \alpha}>\rho_{ \pm} .
\end{aligned}
$$

From (26), we can derive that $\lim _{\alpha \rightarrow 0} \rho_{* \alpha}=+\infty$ and $\lim _{\alpha \longrightarrow 0} \alpha \rho_{* \alpha}=\rho_{+} \rho_{-}\left(u_{-}-u_{+}\right)^{2} / A\left(\sqrt{\rho_{+}}+\sqrt{\rho_{-}}\right)^{2}$. From the above analysis, we can obtain Lemma 2 as follows.

Lemma 2. As $u_{-}>u_{+}$and $\left(u_{+}, \rho_{+}\right) \in S_{1}^{\alpha} S_{2}^{\alpha}\left(u_{-}, \rho_{-}\right)$, $\lim _{\alpha \rightarrow 0} \rho_{* \alpha}=+\infty$ and $\lim _{\alpha \rightarrow 0} \alpha \rho_{* \alpha}=\rho_{+} \rho_{-}\left(u_{-}-u_{+}\right)^{2} /$ $A\left(\sqrt{\rho_{+}}+\sqrt{\rho_{-}}\right)^{2}$, i.e., the phenomenon of concentration occurs.

Next, we consider the formation of delta shock waves. Firstly, we analyze the velocity of intermediate state $u_{*}$. It follows from (3), (24) and Lemma 2 that

$$
\begin{aligned}
\lim _{\alpha \longrightarrow 0} u_{* \alpha} & =\lim _{\alpha \longrightarrow 0}\left(v_{* \alpha}+\beta t\right) \\
& =\lim _{\alpha \longrightarrow 0}\left(u_{-}-h\left(\rho_{* \alpha}, \rho_{-}, \alpha\right)\left(\rho_{* \alpha}-\rho_{-}\right)\right)+\beta t \\
& =u_{\delta}(t) .
\end{aligned}
$$

As the pressure vanishes, from the second equation of (24)(25), the limits of the speeds of $S_{1}^{\alpha}$ and $S_{2}^{\alpha}$ are as follows:

$$
\lim _{\alpha \rightarrow 0} \sigma_{1}^{\alpha}(t)=\lim _{\alpha \longrightarrow 0} \sigma_{2}^{\alpha}(t)=u_{\delta}(t) .
$$




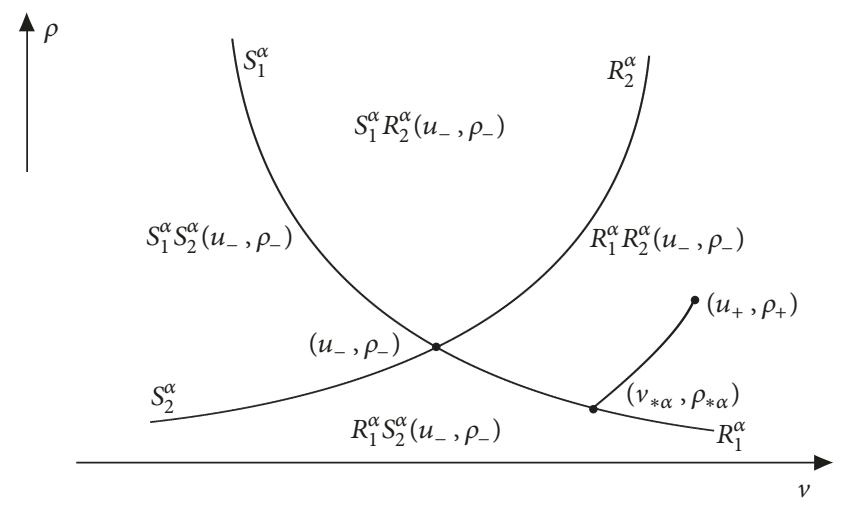

(a) The phase plane

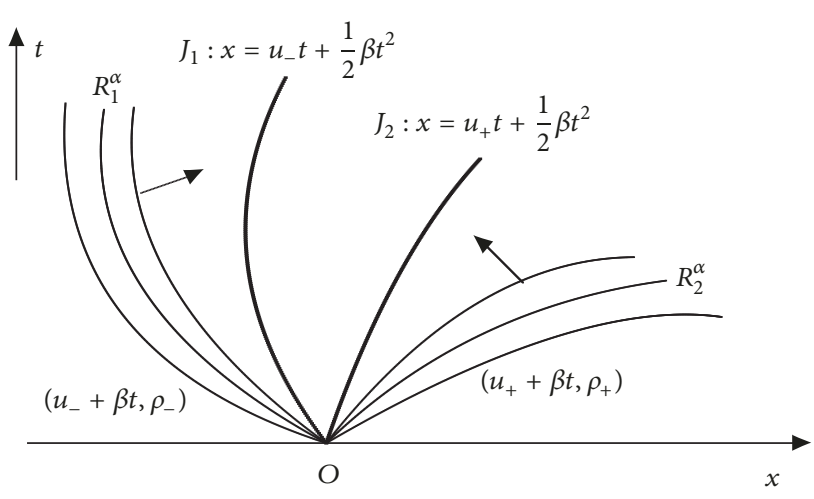

(b) The $(x, t)$ plane

FigURE 3: Riemann solution when $\left(u_{+}, \rho_{+}\right) \in R_{1}^{\alpha} R_{2}^{\alpha}\left(u_{-}, \rho_{-}\right)$.

Equation (27)-(28) shows the two shock waves $S_{1}^{\alpha}$ and $S_{2}^{\alpha}$ are merged into one shock wave with speed $u_{\delta}(t)$ (see Figure 2(b)).

By using the first equation of Rankine-Hugoniot conditions (10) for both $S_{1}^{\alpha}$ and $S_{2}^{\alpha}$, we find

$$
\begin{aligned}
& \sigma_{1}^{\alpha}(t)\left(\rho_{* \alpha}-\rho_{-}\right)=\rho_{* \alpha}\left(v_{* \alpha}+\beta t\right)-\rho_{-}\left(u_{-}+\beta t\right), \\
& \sigma_{2}^{\alpha}(t)\left(\rho_{+}-\rho_{* \alpha}\right)=\rho_{+}\left(u_{+}+\beta t\right)-\rho_{* \alpha}\left(v_{* \alpha}+\beta t\right) .
\end{aligned}
$$

Considering (28)-(29), we obtain

$$
\lim _{\alpha \rightarrow 0}\left(\sigma_{2}^{\alpha}(t)-\sigma_{1}^{\alpha}(t)\right) \rho_{* \alpha}=u_{\delta}(t)[\rho]-[\rho(v+\beta t)] .
$$

From (30), we obtain that

$$
\begin{aligned}
\lim _{\alpha \rightarrow 0} \int_{\sigma_{1}(t) t}^{\sigma_{2}(t) t} \rho_{* \alpha} d x & =\lim _{\alpha \longrightarrow 0}\left(\sigma_{2}^{\alpha}(t)-\sigma_{1}^{\alpha}(t)\right) \rho_{* \alpha} t \\
& =\left(u_{\delta}(t)[\rho]-[\rho(v+\beta t)]\right) t \\
& =\omega(t) .
\end{aligned}
$$

From above analysis and Lemma 2, we have Theorem 3.

Theorem 3. For $u_{-}>u_{+}$, as $\alpha \longrightarrow 0$, the two shock waves of system (1)-(2) converge to a delta shock solution which is the corresponding Riemann solution of system (4).

We have considered the convergence of the Riemann solutions to system (1)-(2) for $u_{-}>u_{+}$as the pressure vanishes. In the following, we study the convergence of the Riemann solutions to system (1)-(2) for $u_{-} \leq u_{+}$.

3.2. The Occurrence of Vacuum as the Pressure Vanishes. In this subcase, as the pressure vanishes, we study the occurrence of vacuum when $\left(u_{+}, \rho_{+}\right) \in R_{1}^{\alpha} R_{2}^{\alpha}\left(u_{-}, \rho_{-}\right)$and $u_{-}<u_{+}$(see Figure 3(a)).

Suppose that $\left(u_{+}, \rho_{+}\right) \in R_{1}^{\alpha} R_{2}^{\alpha}\left(u_{-}, \rho_{-}\right)$, from (7)-(8) and (9), one can derive that

$$
\begin{array}{ll}
u_{+}>u_{-}-g\left(\rho_{-}, \rho_{+}, \alpha\right), & \rho_{+}<\rho_{-}, \\
u_{+}>u_{-}+g\left(\rho_{-}, \rho_{+}, \alpha\right), & \rho_{+}>\rho_{-} .
\end{array}
$$

According to (32)-(33), we obtain that

$$
\alpha<\left(\frac{u_{+}-u_{-}}{f\left(\rho_{+}, 1\right)+f\left(\rho_{-}, 1\right)+k\left(\rho_{+}, \rho_{-}\right)}\right)^{2},
$$

where $k\left(\rho_{+}, \rho_{-}\right)=\ln \left(\left(\sqrt{A \rho_{+}^{2}+B}+\sqrt{A} \rho_{+}\right) /\left(\sqrt{A \rho_{-}^{2}+B}+\right.\right.$ $\left.\left.\sqrt{A} \rho_{-}\right)\right)$. Letting $\alpha_{0}=\left(\left(u_{+}-u_{-}\right) /\left(f\left(\rho_{+}, 1\right)+f\left(\rho_{-}, 1\right)+k\left(\rho_{+}\right.\right.\right.$, $\left.\left.\left.\rho_{-}\right)\right)\right)^{2}$, the right state $\left(u_{+}, \rho_{+}\right) \in R_{1}^{\alpha} R_{2}^{\alpha}\left(u_{-}, \rho_{-}\right)$as $0<\alpha<\alpha_{0}$. Next, we analyze the formation of vacuum when $\left(u_{+}, \rho_{+}\right) \in$ $R_{1}^{\alpha} R_{2}^{\alpha}\left(u_{-}, \rho_{-}\right)$.

Lemma 4. As $u_{+}>u_{-}$and $\left(u_{+}, \rho_{+}\right) \in R_{1}^{\alpha} R_{2}^{\alpha}\left(u_{-}, \rho_{-}\right)$, $\lim _{\alpha \rightarrow 0} \rho_{* \alpha}=0$, i.e., the phenomenon of vacuum occurs.

Proof. From the above analysis, there exists a constant $\alpha_{0}$, such that $\left(u_{+}, \rho_{+}\right) \in R_{1}^{\alpha} R_{2}^{\alpha}\left(u_{-}, \rho_{-}\right)$when $\alpha \in\left(0, \alpha_{0}\right)$. From (7)-(8), we obtain that $v=u_{-}-g\left(\rho_{-}, \rho, \alpha\right)$ on $R_{1}^{\alpha}\left(\rho_{-}, u_{-}\right)$and $v=u_{+}+g\left(\rho_{+}, \rho, \alpha\right)$ on $R_{2}^{\alpha}\left(\rho_{+}, u_{+}\right)$, which derive that

$$
u_{-}-u_{+}=g\left(\rho_{+}, \rho, \alpha\right)+g\left(\rho_{-}, \rho, \alpha\right) \text {. }
$$

If $\lim _{\alpha \rightarrow 0} \rho_{* \alpha}=c_{0} \in\left(0, \min \left(\rho_{-}, \rho_{+}\right)\right)$, as $\alpha$ tends to zero, from (35), we have $u_{-}=u_{+}$, which leads to a contradiction. Therefore, $\lim _{\alpha \rightarrow 0} \rho_{* \alpha}=0$, which means the vacuum occurs as the pressure vanishes.

In Sections 3.1 and 3.2, we discussed the concentration and delta shock wave and the occurrence of vacuum. In the following, we consider the formation of discontinuity.

3.3. The Formation of Discontinuity as the Pressure Vanishes. In this subsection, we are concerned with the formation of discontinuity for $u_{-} \leq u_{+}$, as $\alpha \longrightarrow 0$. Our argument is divided into three parts.

Case 1. $J_{1}+V a c+J_{2}$ for $u_{-}<u_{+}$and $\alpha \longrightarrow 0$.

Lemma 4 shows that the phenomenon of vacuum occurs as $u_{-}<u_{+}$and $\alpha \longrightarrow 0$. Next, we introduce the formation of discontinuity for $u_{-}<u_{+}$. 


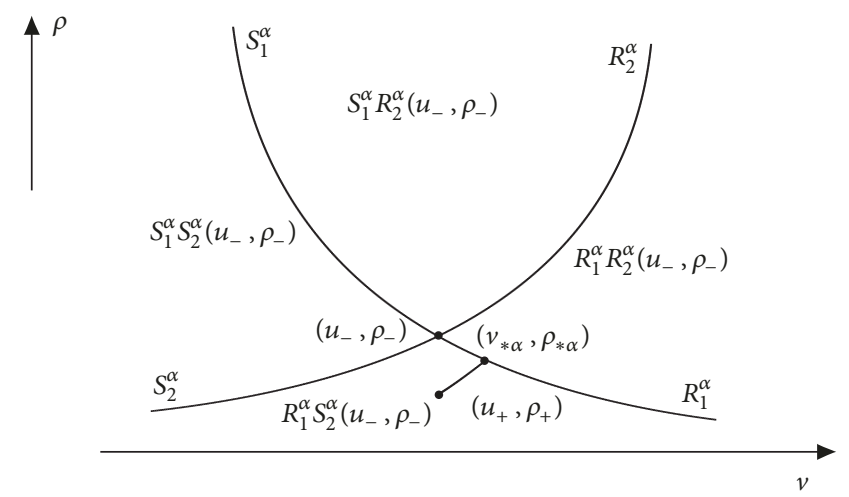

(a) The phase plane

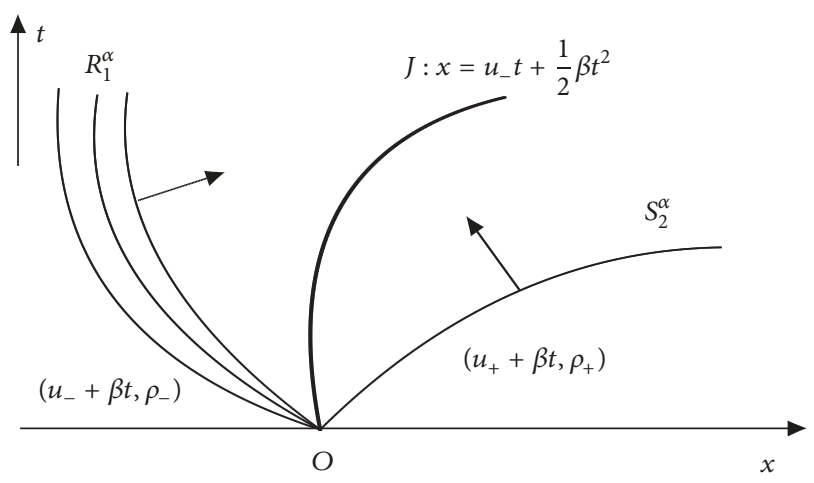

(b) The $(x, t)$ plane

FIGURE 4: Riemann solution when $\left(u_{+}, \rho_{+}\right) \in R_{1}^{\alpha} S_{2}^{\alpha}\left(u_{-}, \rho_{-}\right)$.

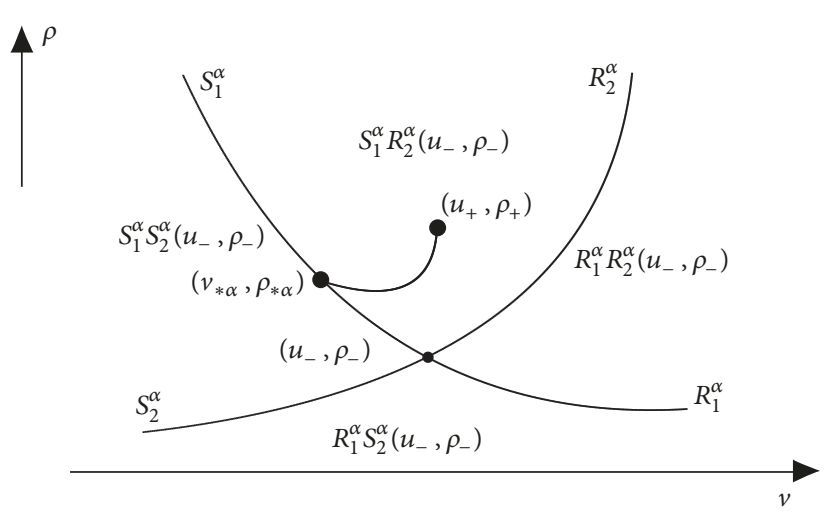

(a) The phase plane

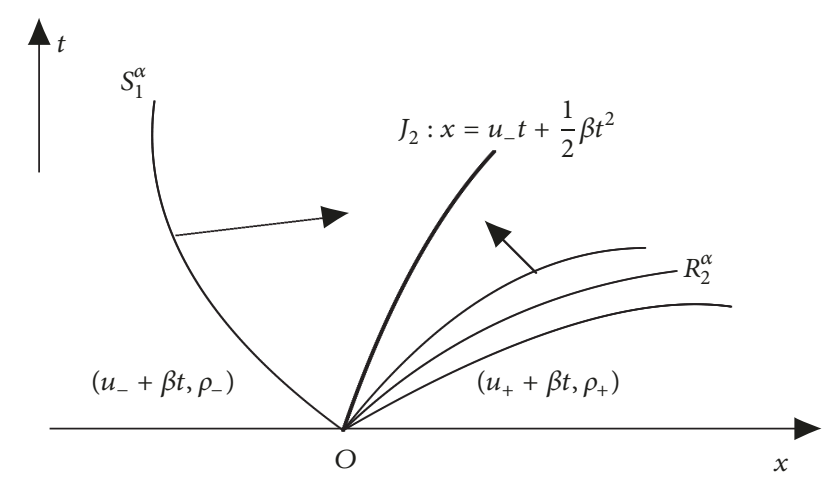

(b) The $(x, t)$ plane

FIGURE 5: Riemann solution when $\left(u_{+}, \rho_{+}\right) \in S_{1}^{\alpha} R_{2}^{\alpha}\left(u_{-}, \rho_{-}\right)$.

When $\left(u_{+}, \rho_{+}\right) \in R_{1}^{\alpha} R_{2}^{\alpha}\left(u_{-}, \rho_{-}\right)$, from (7)-(8), we have

$$
\begin{aligned}
& \lim _{\alpha \longrightarrow 0} v=u_{-} \quad \text { on } R_{1}^{\alpha}\left(\rho_{-}, u_{-}\right), \\
& \lim _{\alpha \longrightarrow 0} v=u_{+} \quad \text { on } R_{2}^{\alpha}\left(\rho_{+}, u_{+}\right)
\end{aligned}
$$

and

$$
\begin{aligned}
& \lim _{\alpha \longrightarrow 0} \lambda_{1}=u_{-}+\beta t, \\
& \lim _{\alpha \longrightarrow 0} \lambda_{2}=u_{+}+\beta t .
\end{aligned}
$$

It follows from (36)-(37) that the two rarefaction waves $R_{1}^{\alpha}$ and $R_{2}^{\alpha}$ turn into two contact discontinuities as the pressure vanishes for $u_{-}<u_{+}$(see Figure 3(b)). We summarize our conclusion as follows.

Theorem 5. For $u_{-}<u_{+}$, as $\alpha \longrightarrow 0$, the two rarefaction waves of system (1)-(2) converge to two contact discontinuities which are the corresponding Riemann solution of system (4).

Case 2. $J^{\star}$ for $u_{-}=u_{+}$and $\alpha \longrightarrow 0$.

We have studied the convergence of the Riemann solution to system (1)-(2) for $u_{-}>u_{+}$and $u_{-}<u_{+}$as the pressure vanishes. Next, we discuss the situation for $u_{-}=$ $u_{+}$. We divide our discussion into two parts for $\left(u_{+}, \rho_{+}\right) \in$ $R_{1}^{\alpha} S_{2}^{\alpha}\left(u_{-}, \rho_{-}\right)$(see Figure $\left.4(\mathrm{a})\right)$ and $\left(u_{+}, \rho_{+}\right) \in S_{1}^{\alpha} R_{2}^{\alpha}\left(u_{-}, \rho_{-}\right)$ (see Figure 5(a)).

When $\left(u_{+}, \rho_{+}\right) \in R_{1}^{\alpha} S_{2}^{\alpha}\left(u_{-}, \rho_{-}\right)$, from (7) and (25), we deduce that

$$
\lim _{\alpha \longrightarrow 0} v_{* \alpha}+\beta t=\lim _{\alpha \longrightarrow 0} \lambda_{1}\left(v_{* \alpha}, \rho_{* \alpha}\right)=\sigma_{2}^{\alpha}(t)=u_{-}+\beta t,
$$

which implies that the rarefaction wave $R_{1}\left(\rho_{-}, u_{-}\right)$and the shock wave $S_{2}\left(\rho_{+}, u_{+}\right)$become a contact discontinuity as the pressure vanishes (see Figure 4(b)).

Similarly, from (8) and (24), we know that

$$
\lim _{\alpha \longrightarrow 0} v_{* \alpha}+\beta t=\lim _{\alpha \longrightarrow 0} \lambda_{2}\left(v_{* \alpha}, \rho_{* \alpha}\right)=\sigma_{1}^{\alpha}(t)=u_{-}+\beta t,
$$

which means that the rarefaction wave $R_{2}^{\alpha}\left(\rho_{+}, u_{+}\right)$and the shock wave $S_{1}^{\alpha}\left(\rho_{-}, u_{-}\right)$turn into a contact discontinuity as $\alpha \longrightarrow 0$ (see Figure 5(b)).

We have considered the convergence of the Riemann solution of system (1)-(2) for $u_{-}=u_{+}$, as the pressure vanishes. We summarize our main results as follows.

Theorem 6. For $u_{-}=u_{+}$, as $\alpha \longrightarrow 0$, the Riemann solutions of system (1)-(2) converge to a contact discontinuity $J^{\star}$ which is the corresponding Riemann solution of system (4). 


\section{Conclusion}

We have considered the convergence of the Riemann solutions to the modified Chaplygin gas equations with a coulomb-like friction term. As the pressure vanishes, the concentration and delta shock wave are concerned. Meanwhile the occurrence of vacuum and formation of discontinuity are studied. We find that the Riemann solutions of (1)-(2) converge to the corresponding Riemann solutions of (4) as the pressure vanishes.

\section{Data Availability}

No data were used to support this study.

\section{Conflicts of Interest}

The authors declare that they have no conflicts of interest.

\section{Acknowledgments}

This work is partially supported by the Natural Science Foundation of Xinjiang (Grant no. 2017D01C053).

\section{References}

[1] H. B. Benaoum, "Accelerated universe from modified Chaplygin gas and tachyonic fluid," Physics, 2002.

[2] U. Debnath, "Constraining the parameters of modified chaplygin gas in einstein-aether gravity," Advances in High Energy Physics, vol. 2014, Article ID 653630, 8 pages, 2014.

[3] B. C. Paul, P. Thakur, and A. Saha, "Modified Chaplygin gas as scalar field and holographic dark energy model," High Energy Physics-Theory, 2008.

[4] S. Chakraborty and U. Debnath, "Role of a chameleon field in the presence of variable modified Chaplygin gas in Brans-Dicke theory," Canadian Journal of Physics, vol. 90, no. 2, pp. 131-135, 2012.

[5] J. Zschoche, "The Chaplygin gas equation of state for the quantized free scalar field on cosmological spacetimes," Annales Henri Poincare. A Journal of Theoretical and Mathematical Physics, vol. 15, no. 7, pp. 1285-1325, 2014.

[6] M. Szydłowski and W. Czaja, "Toward reconstruction of the dynamics of the Universe from distant type Ia supernovae," Physical Review D: Particles, Fields, Gravitation and Cosmology, vol. 69 , no. 8, pp. 281-317, 2004.

[7] H. B. Benaoum, "Modified Chaplygin gas cosmology," Advances in High Energy Physics, vol. 2012, Article ID 357802, 12 pages, 2012.

[8] S. Bhattacharya and U. Debnath, "Thermodynamics of Modified Chaplygin Gas and Tachyonic Field," International Journal of Theoretical Physics, vol. 51, no. 2, pp. 565-576, 2012.

[9] S. Chattopadhyay and U. Debnath, "Interaction between phantom field and modified Chaplygin gas," Astrophysics and Space Science, vol. 326, no. 2, pp. 155-158, 2010.

[10] S. S. E. Costa, "Relations between the modified Chaplygin gas and a scalar field," Physics, 2008.

[11] E. O. Kahya and B. Pourhassan, "Observational constraints on the extended Chaplygin gas inflation," Astrophysics and Space Science, vol. 353, no. 2, pp. 677-682, 2014.
[12] P. Rudra, C. Ranjit, and S. Kundu, "How effective is new variable modified Chaplygin gas to play the role of dark energya dynamical system analysis in RS II brane model," Astrophysics and Space Science, vol. 347, no. 2, pp. 433-444, 2013.

[13] H. Yang and J. Wang, "Delta-shocks and vacuum states in the vanishing pressure limit of solutions to the isentropic Euler equations for modified Chaplygin gas," Journal of Mathematical Analysis and Applications, vol. 413, no. 2, pp. 800-820, 2014.

[14] S. A. Chaplygin, "On gas jets," Scientific Memoirs, Moscow University Mathematic Physics, vol. 21, no. 1063, pp. 1-121, 1904.

[15] H.-S. Tsien, "Two-dimensional subsonic flow of compressible fluids," Journal of the Aeronautical Sciences, vol. 6, pp. 399-407, 1939.

[16] T. von Karman, "Compressibility effects in aerodynamics," Journal of the Aeronautical Sciences, vol. 8, pp. 337-356, 1941.

[17] Y. Brenier, "Solutions with concentration to the Riemann problem for the one-dimensional Chaplygin gas equations," Journal of Mathematical Fluid Mechanics, vol. 7, no. suppl. 3, pp. S326-S331, 2005.

[18] L. Guo, W. Sheng, and T. Zhang, "The two-dimensional Riemann problem for isentropic Chaplygin gas dynamic system," Communications on Pure and Applied Analysis, vol. 9, no. 2, pp. 431-458, 2010.

[19] D. Kong and C. Wei, "Formation and propagation of singularities in one-dimensional Chaplygin gas," Journal of North China Institute of Science \& Technology, vol. 80, pp. 58-70, 2014.

[20] G. Lai, W. Sheng, and Y. Zheng, "Simple waves and pressure delta waves for a Chaplygin gas in two-dimensions," Discrete and Continuous Dynamical Systems - Series A, vol. 31, no. 2, pp. 489-523, 2011.

[21] S. Chen and A. Qu, "Two-dimensional Riemann problems for Chaplygin gas," SIAM Journal on Mathematical Analysis, vol. 44, no. 3, pp. 2146-2178, 2012.

[22] Z. Wang and Q. Zhang, "The Riemann problem with delta initial data for the one-dimensional Chaplygin gas equations," Acta Mathematica Scientia B, vol. 32, no. 3, pp. 825-841, 2012.

[23] G. Wang, B. Chen, and Y. Hu, "The two-dimensional Riemann problem for Chaplygin gas dynamics with three constant states," Journal of Mathematical Analysis \& Applications, vol. 393, pp. 544-562, 2012.

[24] C. Shen, "The Riemann problem for the Chaplygin gas equations with a source term," ZAMM - Journal of Applied Mathematics and Mechanics/Zeitschrift für Angewandte Mathematik und Mechanik, vol. 96, no. 6, pp. 681-695, 2016.

[25] G. Faccanoni and A. Mangeney, "Exact solution for granular flows," International Journal for Numerical and Analytical Methods in Geomechanics, vol. 37, no. 10, pp. 1408-1433, 2013.

[26] M. Sun, "The exact Riemann solutions to the generalized Chaplygin gas equations with friction," Communications in Nonlinear Science and Numerical Simulation, vol. 36, pp. 342353, 2016.

[27] L. Guo, T. Li, L. Pan, and X. Han, "The Riemann problem with delta initial data for the one-dimensional Chaplygin gas equations with a source term," Nonlinear Analysis: Real World Applications, vol. 41, pp. 588-606, 2018.

[28] C. Shen, "The Riemann problem for the pressureless Euler system with the Coulomb-like friction term," IMA Journal of Applied Mathematics, vol. 81, no. 1, pp. 76-99, 2016.

[29] D. A. Daw and M. Nedeljkov, "Shadow waves for pressureless gas balance laws," Applied Mathematics Letters, vol. 57, pp. 5459, 2016. 
[30] J. Li, "Note on the compressible Euler equations with zero temperature," Applied Mathematics Letters, vol. 14, no. 4, pp. 519-523, 2001.

[31] G. Chen and H. Liu, "Formation of delta-shocks and vacuum states in the vanishing pressure limit of solutions to the Euler equations for isentropic fluids," Journal of Speech \& Hearing Research, vol. 28, pp. 445-448, 2006.

[32] G.-Q. Chen and H. Liu, "Concentration and cavitation in the vanishing pressure limit of solutions to the Euler equations for nonisentropic fluids," Physica D: Nonlinear Phenomena, vol. 189, no. 1-2, pp. 141-165, 2004.

[33] C. Shen, "The limits of Riemann solutions to the isentropic magnetogasdynamics," Applied Mathematics Letters, vol. 24, no. 7, pp. 1124-1129, 2011.

[34] C. Shen and M. Sun, "Formation of delta shocks and vacuum states in the vanishing pressure limit of Riemann solutions to the perturbed Aw-RAScle model," Journal of Differential Equations, vol. 249, no. 12, pp. 3024-3051, 2010.

[35] W. Sheng, G. Wang, and G. Yin, "Delta wave and vacuum state for generalized Chaplygin gas dynamics system as pressure vanishes," Nonlinear Analysis: Real World Applications, vol. 22, pp. 115-128, 2015.

[36] G. Yin and W. Sheng, "Delta shocks and vacuum states in vanishing pressure limits of solutions to the relativistic Euler equations for polytropic gases," Journal of Mathematical Analysis and Applications, vol. 355, no. 2, pp. 594-605, 2009.

[37] L. Guo, T. Li, and G. Yin, "The vanishing pressure limits of Riemann solutions to the Chaplygin gas equations with a source term," Communications on Pure and Applied Analysis, vol. 16, no. 1, pp. 295-309, 2017.

[38] L. Guo, T. Li, and G. Yin, "The limit behavior of the Riemann solutions to the generalized Chaplygin gas equations with a source term," Journal of Mathematical Analysis and Applications, vol. 455, no. 1, pp. 127-140, 2017.

[39] C. Shen and M. Sun, "A distributional product approach to the delta shock wave solution for the one-dimensional zeropressure gas dynamics system," International Journal of NonLinear Mechanics, 8 pages, 2018.

[40] M. Sun, "Singular solutions to the Riemann problem for a macroscopic production model," ZAMM - Journal of Applied Mathematics and Mechanics/Zeitschrift für Angewandte Mathematik und Mechanik, vol. 97, no. 8, pp. 916-931, 2017. 


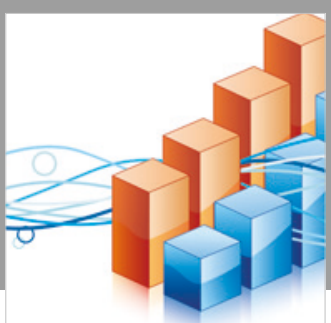

Advances in

Operations Research

\section{-n-m}
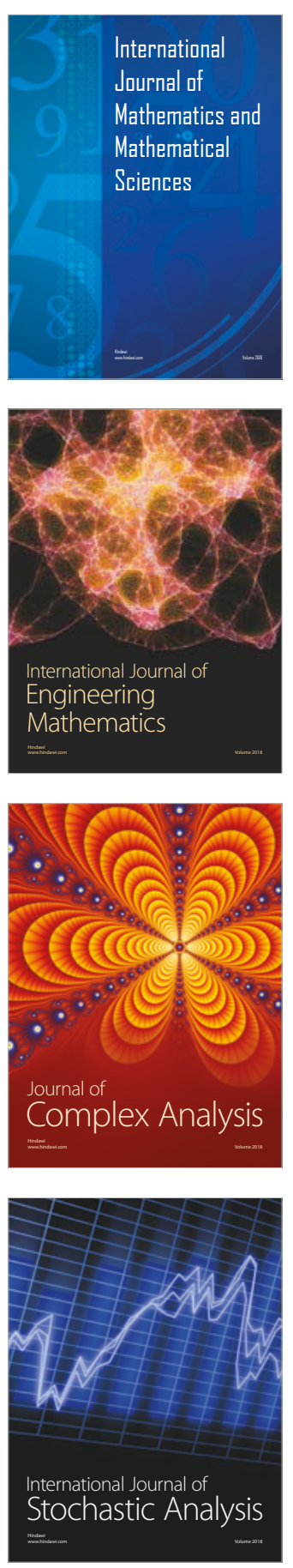
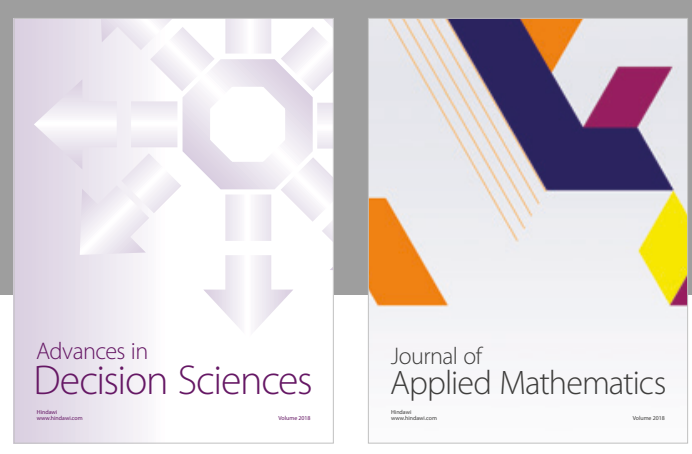

Journal of

Applied Mathematics
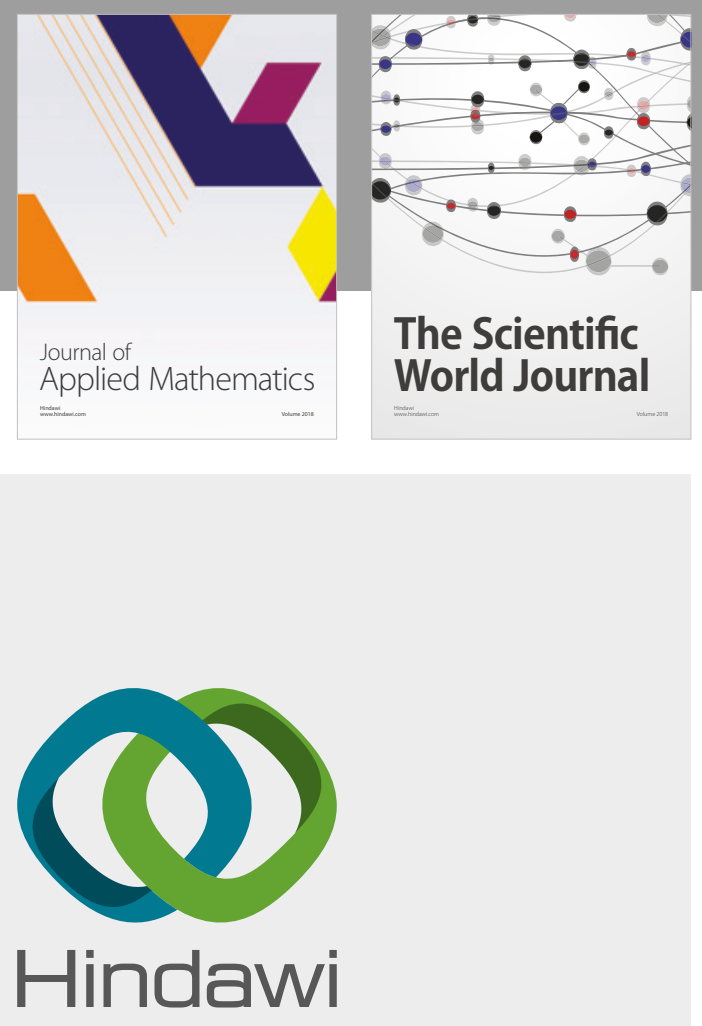

Submit your manuscripts at

www.hindawi.com

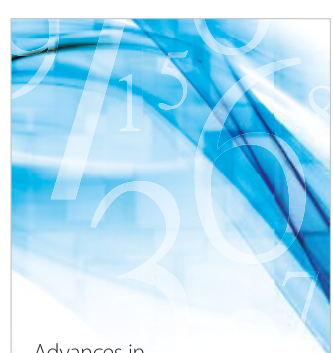

Advances in
Numerical Analysis
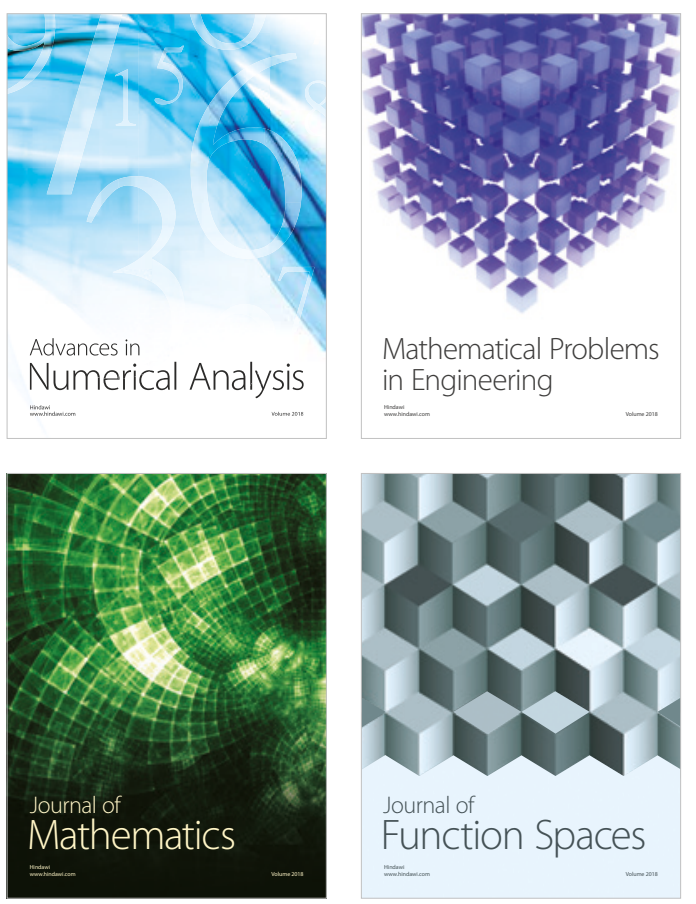

Mathematical Problems in Engineering

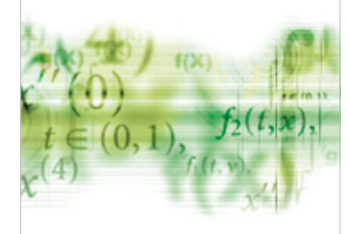

International Journal of

Differential Equations

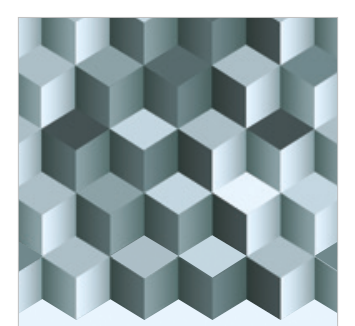

Journal of

Function Spaces

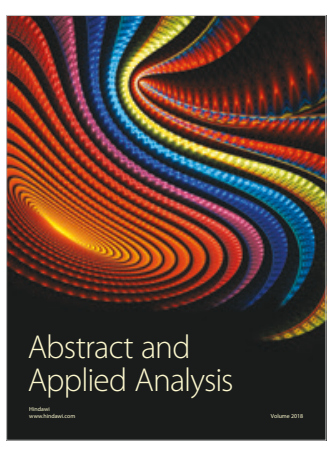

The Scientific

World Journal

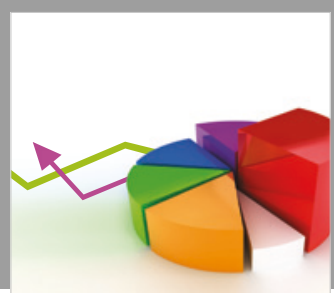

Journal of

Probability and Statistics
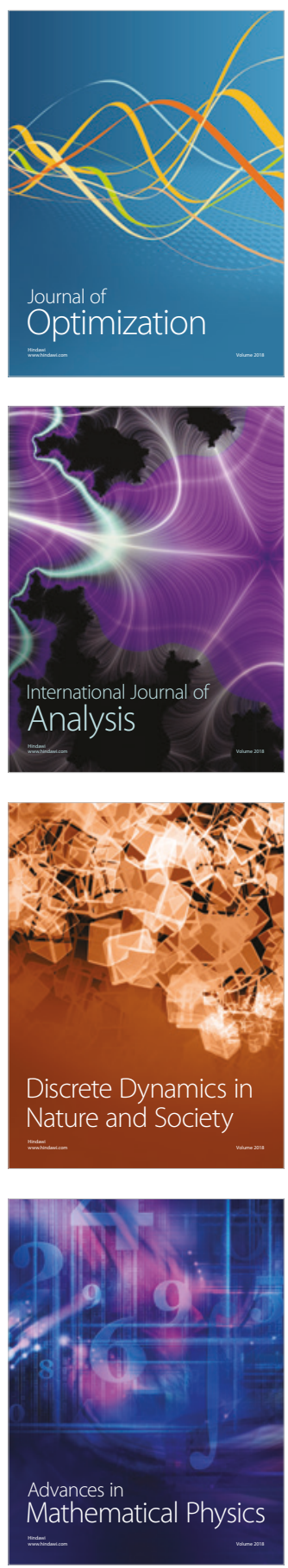